\title{
Construction of a Plant Binary Expression Vector for the Potato Host Factor Genes StTOM1 and StTOM3
}

\author{
Jingjing Song \\ Qinzhou University, Qinzhou 535011, China \\ Guangxi University, Nanning 530004, China
}

\begin{abstract}
In this article, we designed PCR primers according to the cDNA sequence of potato host factor genes StTOM1 and StTOM3 and the restriction enzyme sites of the intermediate vector pUCCRNAi to gain two RNA interference segments, which length were 272bp and 182bp, and then connected to the vector pUCCRNAi from two directions simultaneously. After the validation of sequencing and digestion, the bidirectional RNA interference segment with intron was digested from pUStT1-StT3-dRi $( \pm)$ and connected to pBI121, thus the plant binary expression vector pBIStT1-StT3-dRi $( \pm)$ was constructed.
\end{abstract}

Keywords: Potato, StTOM1, StTOM3, Plant binary expression vector.

\section{Introduction}

Potato is the world's fourth most-widely-consumed crop, the development of potato industry is severely restricted by viral diseases as its reproduction through tubers facilitates the spreading of viruses in potato seed, and causes the deterioration of potato seed. For this reason, it appears very important to cultivate the anti-viral disease potato.

As intracellular obligatory parasites, viruses relied on DNA or RNA segments to encode, replicate, infect and spread the required genetic information. For its simple structure, a virus needs to complete the cycle of infection with the help of host factor. Host factor refers to the host protein and structure supporting the replication of virus and its transportation inside host (Huang et al., 2010). In recent years, some host factor genes have been discovered gradually and proved to support the replication of viruses. For instance, AtTOM1 discovered in arabidopsis thaliana was reported to support the replication of tobacco mosaic virus (TMV), cucumber mosaic virus (CMV) and tomato mosaic virus (ToMV) (Hagiwara-Komoda et al., 2008). It was also proved in some studies that when the genes TOM1 and TOM3 were knocked out simultaneously in both arabidopsis thaliana and tobacco, the replication of TMV was inhibited (Asano et al., 2005). With all these evidences, it is proved that TOM1 and TOM3 are the host factors for replication of viruses in plants. Additionally, some other host factors have been also discovered in plants, including RTM1 and RTM2 (Chisholm et al., 2001), LSM1 and LSM6 (Noueiry and Ahlquist, 2003), THH1 (Fujisaki et al., 2006), TOM2A (Fujisaki and Ishikawa, 2008), CUM1 and CUM2 (Roudet-Tavert et al., 2007) and pectin methylesterase (PME) (Micheli, 2001). Now, few studies have focused on the information and acting mechanism of these host factors.

\section{Result and Analysis}

\subsection{PCR Amplification of Interference Segment of Potato Genes StTOM1and StTOM3}

PCR amplification was conducted to gain StTOM1 and StTOM3 interference segments by taking StTOM1-dRi-F /StTOM1-dRi-R and StTOM3-dRi-F /StTOM3-dRi-R as primer respectively, and the cDNA cloning of StTOM1 and StTOM3 as the template. For gene StTOM1, the expected length of interference segment was 272bp. For StTOM3, it was 182bp. The stripes of gene obtained through amplification were clear, and had expected size (Fig. 1). 


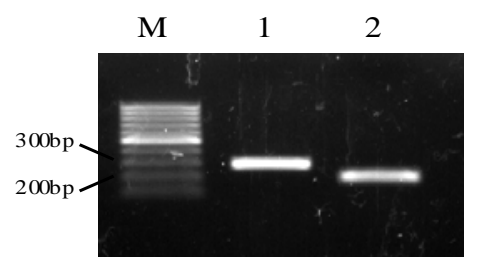

Fig.1 PCR result of target fragment

Note: M: GeneRuler ${ }^{\mathrm{TM}}$ 100bp DNA Ladder; 1: PCR amplification of RNA interference fragment of StTOM1; 2: PCR amplification of RNA interference fragment of StTOM3

\subsection{Construction and Identification of Intermediate Vector for Potato Genes StTOM1 and StTOM3}

By digestion, StTOM1 RNAi segment and StTOM3 RNAi segment were connected to pUCCRNAi plasmid resistant to ampicillin, so as to gain intermediate reverse vector pUStT1-StT3-dRi(-). Subsequently, pUStT1-StT3-dRi(-) was digested with Pst I to obtain 660bp segment, which was preliminarily validated as correct clone (Fig. 2A). After validation by sequencing, StTOM1 and StTOM3 were connected after being digested with Spe I and Bgl II, and forward connected to the vector pUStT1-StT3-dRi(-) digested with BamH I and Xba I, so as to obtain the duplex connected clone. For validation, 1,100bp stripe was obtained by digestion with Xho I and Sal I (Fig. 2B). The stripe had the size matching with the target segment, proving that the bidirectional StTOM1StTOM3-dRNAi segment had been successfully connected to intermediate vector. This successfully constructed intermediate vector was named pUStT1-StT3-dRi $( \pm)$. The process of construction is detailed in Fig. 3.

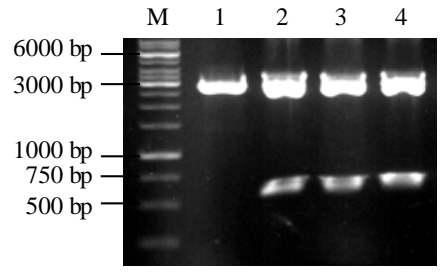

A

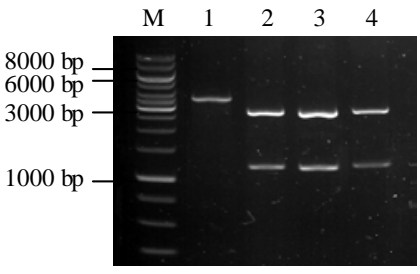

B

Fig.2 Verification of the intermediate vector pUStT1-StT3-dRi $( \pm)$ by digestion

Note: A: Identification of the intermediate reverse vector pUStT1-StT3-dRi( - ) by digestion; M: GeneRuler ${ }^{\text {TM }} 1 \mathrm{~kb}$ DNA Ladder; 1 : Plasmid pUCCRNAi digested with Pst I (Negative control); 2 4: Vector pUStT1-StT3-dRi( - ) digested with Pst I; B: Identification of the duplex connected vector pUStT1-StT3-dRi $( \pm)$ by digestion; M: GeneRuler ${ }^{\mathrm{TM}} 1 \mathrm{~kb}$ DNA Ladder; 1 : Plasmid pUCCRNAi digested by Xho I and Sal I (Negative control); 2 4: Vector pUStT1-StT3-dRi( \pm ) digested by Xho I and Sal I

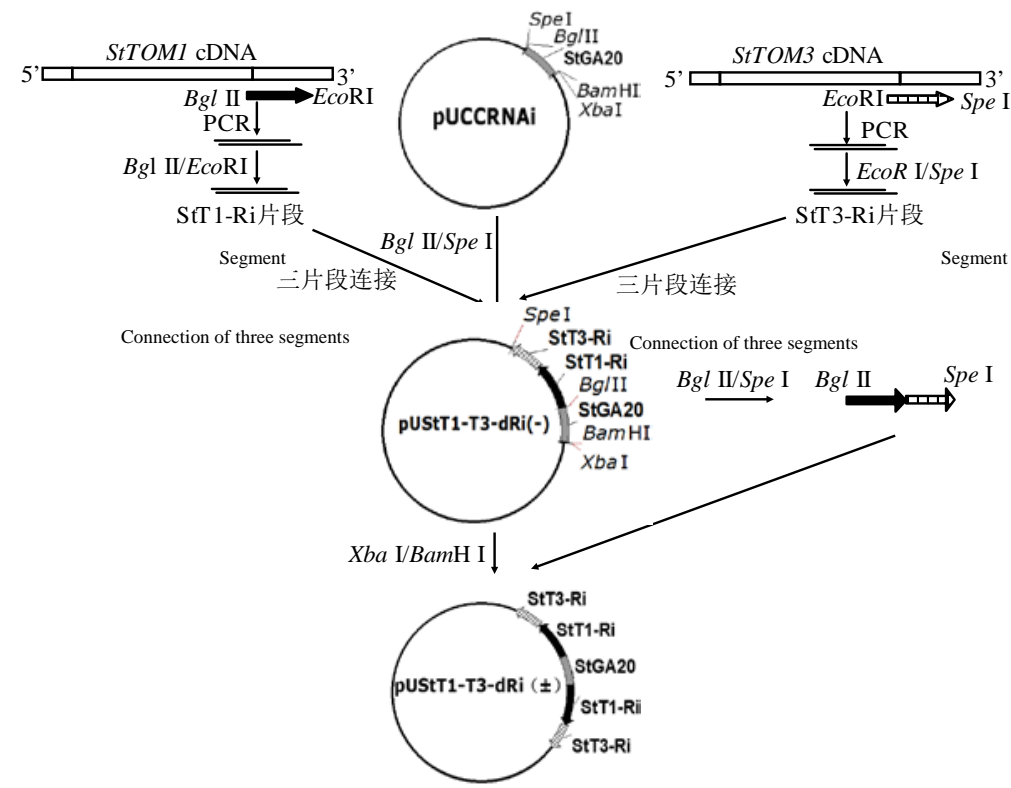

Fig.3 Construction of the intermediate vector pUStT1-StT3-dRi $( \pm)$ 


\subsection{Construction and Identification of Plant Binary Expression Vector for Potato Genes StTOM1and StTOM3}

Based on the characteristics of restriction enzyme sites on plasmids pUCCRNAi and pBI121, the constructed bidirectional StTOM1- StTOM3-dRNAi segment containing intron was separated from the intermediate vector pUStT1-StT3-dRi $( \pm)$ by digestion, and connected to the vector pBI121 digested with $X b a$ I and Sma I, so as to gain the plant binary expression vector pBIStT1-StT3-dRi $( \pm)$. Through validation by digestion with $\mathrm{Bgl}$ II, the plasmid pBI121 had two Bgl II enzyme sites, so two segments could be taken from the plasmid by digestion with $\mathrm{Bgl}$ II, i.e. one 8,000bp segment and one 7,000bp segment. Meanwhile, a Bgl II enzyme site was transferred when transforming the alien segment into the constructed vector. Hence, one 8,000bp segment and two 4,000bp segments could be gained by digestion with $\mathrm{Bgl}$ II. The results were presented in Fig. 4A, and had the same size as target segment, proving that the plant binary expression vector pBIStT1-StT3-dRi $( \pm)$ was successfully constructed. The process of construction was presented in Fig. 5.

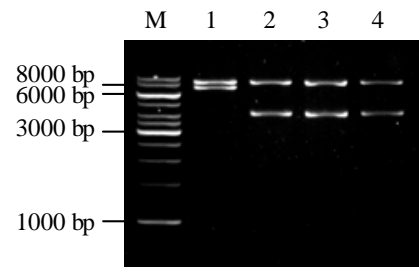

A

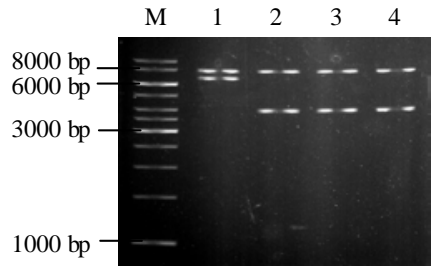

B

Fig.4 Verification of the plant binary expression vector pBIStT1-StT3-dRi( \pm ) in E.coli DH5 $\alpha$ and Agrobacterium EHA105 by digestion

Note: A: Verification of the vector pBIStT1-StT3-dRi( \pm ) in E.coli DH5 $\alpha$; M: GeneRuler ${ }^{\mathrm{TM}} 1 \mathrm{~kb}$ DNA Ladder; 1 : Plasmid pBI121 digested by Bgl II (Negative control); 2 4: Vector pBIStT1-StT3-dRi $( \pm$ ) digested by Bgl II; B: Verification of the vector pBIStT1-StT3-dRi( \pm ) in EHA105; M: GeneRuler ${ }^{\text {TM }}$ 1kb DNA Ladder; 1: Plasmid pBI121 digested by Bgl II (Negative control); 2 4: Vector pBIStT1-StT3-dRi( \pm ) digested by Bgl II
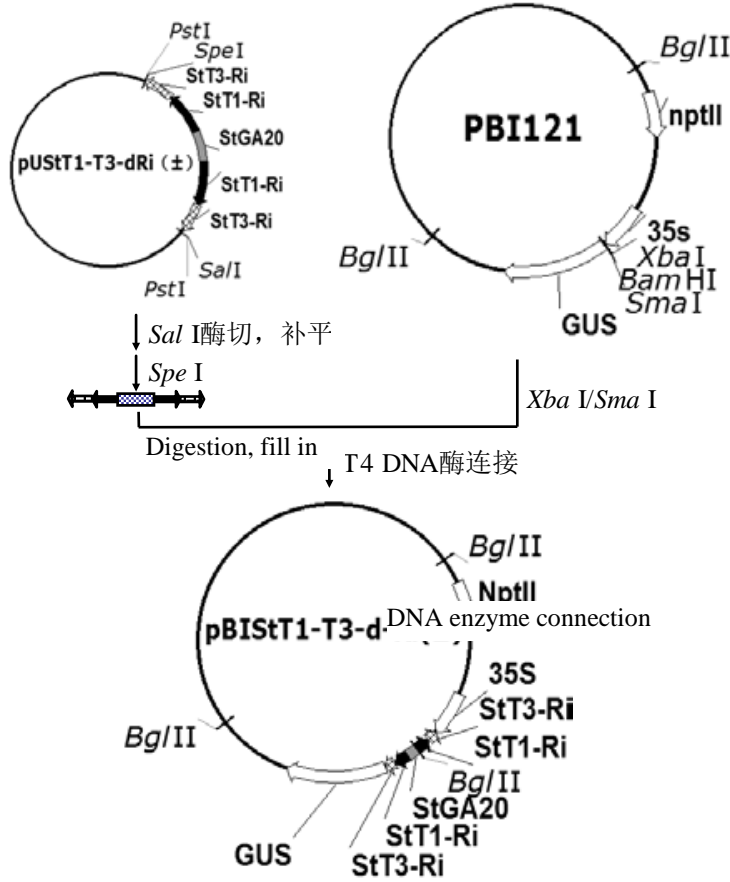

Fig.5 Construction of the plant binary expression vector pBIStT1-StT3-dRi $( \pm)$

\section{Discussion}

Host factors are involved in the infection of viruses, so they can be regulated to prevent the replication and transfer of viruses in host, making it possible to prevent viral diseases. This approach is of great significance to the research, prevention and treatment of viral diseases for humans, animals 
and plants, so it is one of the most promising strategy for anti-viral genetic engineering breeding. As proved in some studies, some hosts could effectively control the occurrence of viral diseases after mutation without affecting their normal growth. For instance, when TOM1 and TOM3 were knocked out in arabidopsis thaliana and tobacco, the replication of TMV was inhibited, but transgenic plants could grow and develop normally (Asano et al., 2005). After mutation of LSM1 and LSM6, the replication level of Bean commom mosaic virus (BMV) was lowered, but mutants still grew and developed as usual (Noueiry and Ahlquist, 2003). It was once reported that AtTHH1 could also inhibit the replication of TMV without affecting the growth of arabidopsis thaliana (Fujisaki et al., 2006). All these studies reveal that it is feasible to construct new anti-viral germplasms.

\section{Material and Method}

\subsection{Design and Synthesis of Primers}

The primer sequences are presented in Table 1. The synthesis of primers was conducted by Sangon Biotech (Shanghai) Co., Ltd.

Table 1 Primers used for PCR

\begin{tabular}{cc}
\hline Primer name & Primer sequence (5’'3’) \\
\hline StTOM1-dRi-F & GGAAGATCTGCATCTCTTGACGTCTTGGACC \\
StTOM1-dRi-R & CGGAATTCGGCCTTTCCACAGAATTATTGC \\
StTOM3-dRi-F & GGAATTCCCTGTTAGTGGAGATACTGCCTTCT \\
StTOM3-dRi-R & CGGACTAGTCAAATCACATCCAGGTAAAAGCC \\
nptII-1F & GGTGGAGAGGCTATTCGGCTATGA \\
nptII-1R & TGATATTCGGCAAGCAGGCATCG \\
35S-1F & CTTACGCAGCAGGTCTCATCAAGA \\
35S-1R & GGCAATGGAATCCGAGGAGGTT \\
\hline
\end{tabular}

\subsection{PCR Amplification of Target Gene}

Taking the cDNA clone of genes StTOM1 and StTOM3 as a template, amplification was carried out with the primers StTOM1-dRi-F /StTOM1-dRi-R and StTOM3-dRi-F /StTOM3-dRi-R. The PCR reaction system was $25 \mu \mathrm{L}: 5 \times$ PrimeSTAR $^{\mathrm{TM}}$ Buffer $5 \mu \mathrm{L}, 2.5 \mathrm{mmol} / \mathrm{L}$ dNTP $2 \mu \mathrm{L}, 1 \mu \mathrm{L}$ each for $10 \mu \mathrm{mol} / \mathrm{L}$ primers, $1 \mu \mathrm{L}$ each for $10 \mathrm{ng} / \mu \mathrm{L}$ templates, PrimeSTAR ${ }^{\mathrm{TM}} \mathrm{HS}$ DNA Polymerase $(2.5 \mathrm{U} / \mu \mathrm{L})$ $0.2 \mu \mathrm{L}$, and $\mathrm{ddH}_{2} \mathrm{O} 14.8 \mu \mathrm{L}$. The reaction conditions were as follows: initial denaturation at $94^{\circ} \mathrm{C}$ for 3min, denaturation at $94^{\circ} \mathrm{C}$ for $30 \mathrm{~s}$, annealing at $61^{\circ} \mathrm{C}$ for 30 s, extension at $72^{\circ} \mathrm{C}$ for $30 \mathrm{~s}$, 30 cycles, and extension at $72^{\circ} \mathrm{C}$ for $10 \mathrm{~min}$. The size and quality of stripes were tested by $1 \%$ agarose gel electrophoresis.

\subsection{Construction of Intermediate Vector pUStT1-StT3-dRi( \pm )}

After purification of PCR product, StTOM1 gene segment was digested with Bgl II and EcoR I, while StTOM3 gene segment was digested with EcoR I and Spe I. Meanwhile, the plasmid pUCCRNAi was digested with Spe I and Bgl II. Through electrophoresis and purification by gel slices, the products from the abovementioned digestions were used to recycle interference segments and vector. Then, StTOM1 RNAi segment, StTOM3 RNAi segment and pUCCRNAi vector were connected at $16^{\circ} \mathrm{C}$ for $8 \mathrm{~h}$ under the effect of T4 DNA ligase, and the product from such connection was used to transform the competent cells of Escherichia coli DH5a, which were screened on the LA culture medium containing 100mg/L Ampicillin. Through the shaking culture of selected single colonies in the LB culture medium containing 50mg/L Ampicillin, plasmids were extracted and validated by digestion with Spe I and Bgl II. From plasmids identified to be positive, 3 were selected and sent to Sangon Biotech (Shanghai) Co., Ltd. for sequencing. 
Based on the results of sequencing, the clone pUStT1-StT3-dRi(-) with the same sequence as the original template was selected, and its plasmids were digested with Spe I and Bgl II and 454bp connected segments StTOM1 and StTOM3 were recycled. Through digestion with BamH I and Xba I, the vector pUStT1-StT3-dRi(-) was recycled. The recycled segment and vector were connected by T4 DNA ligase to transform the Escherichia coli and select the positive clone resistant to Ampicillin through screening. After selecting the positive clone sway and extracting the plasmids, the bidirectional connected vector pUStT1-StT3-dRi $( \pm)$ was digested with Xho I and Sal I to determine whether the size of connected segment was correct.

\subsection{Construction of Plant Binary Expression Vector pBIStT1-StT3-dRi( \pm )}

The constructed plasmid pBIStT1-StT3-dRi $( \pm)$ was digested with Sal I , and then filled in by T4 DNA polymerase. After being deactivated and purified, the product was digested with Spe I, and then recycled by gel slices to obtain 1,108bp interference segment StTOM1- StTOM3-dRNAi. The plasmid pBI121 was digested with Xba I. After purification, it was digested with Sma I. The obtained vector was connected to the interference segment StTOM1- StTOM3-dRNAi through T4 DNA ligase at $16^{\circ} \mathrm{C}$ for $8 \mathrm{~h}$. From the product of such connection, $5 \mu \mathrm{L}$ was taken to transform the competent cells of Escherichia coli DH5 $\alpha$, and then placed in screening on the LA culture medium containing $100 \mathrm{mg} / \mathrm{L}$ Kanamycin. After selecting positive strain sway and extracting plasmids, the interference segment was digested with Bgl II to validate whether it was successfully connected.

\section{References}

[1]. Asano M, Satoh R, Mochizuki A, Tsuda S, Yamanaka T, Nishiguchi M, Hirai K, Meshi T, Naito S, Ishikawa M. 2005. Tobamovirus-resistant tobacco generated by RNA interference directed against host genes. FEBS letters 579(20):4479-4484.

[2]. Banerjee AK, Prat S, Hannapel DJ. 2006. Efficient production of transgenic potato (S. tuberosum L. ssp. andigena) plants via Agrobacterium tumefaciens-mediated transformation. Plant Science 170(4):732-738.

[3]. Chisholm ST, Parra MA, Anderberg RJ, Carrington JC. 2001. Arabidopsis RTM1 and RTM2 genes function in phloem to restrict long-distance movement of tobacco etch virus. Plant Physiology 127(4):1667-1675.

[4]. Fujisaki K, Ishikawa M. 2008. Identification of an Arabidopsis thaliana protein that binds to tomato mosaic virus genomic RNA and inhibits its multiplication. Virology 380(2):402-411.

[5]. Fujisaki K, Ravelo GB, Naito S, Ishikawa M. 2006. Involvement of THH1, an Arabidopsis thaliana homologue of the TOM1 gene, in tobamovirus multiplication. Journal of general virology 87(8):2397-2401.

[6]. Hagiwara-Komoda Y, Hirai K, Mochizuki A, Nishiguchi M, Meshi T, Ishikawa M. 2008. Overexpression of a host factor TOM1 inhibits tomato mosaic virus propagation and suppression of RNA silencing. Virology 376(1):132-139.

[7]. Huang T-S, Wei T, Laliberte J-F, Wang A. 2010. A host RNA helicase-like protein, AtRH8, interacts with the potyviral genome-linked protein, VPg, associates with the virus accumulation complex, and is essential for infection. Plant physiology 152(1):255-266.

[8]. Micheli F. 2001. Pectin methylesterases: cell wall enzymes with important roles in plant physiology. Trends in plant science 6(9):414-419.

[9]. Noueiry AO, Ahlquist P. 2003. Brome mosaic virus RNA replication: revealing the role of the host in RNA virus replication. Annual review of phytopathology 41(1):77-98.

[10]. Roudet-Tavert G, Michon T, Walter J, Delaunay T, Redondo E, Le Gall O. 2007. Central domain of a potyvirus VPg is involved in the interaction with the host translation initiation factor eIF4E and the viral protein HcPro. Journal of general virology 88(3):1029-1033. 